\title{
Single Enzyme Studies Reveal the Existence of Discrete Functional States for Monomeric Enzymes and How They Are "Selected" upon Allosteric Regulation
}

Hatzakis, Nikos S.; Wei, Li; Jørgensen, Sune Klamer; Kunding, Andreas Hjarne; Bolinger, Pierre-Yves; Ehrlich, Nicky; Makarov, Ivan; Skjot, Michael; Svendsen, Allan; Hedegård, Per

Total number of authors:

11

Published in:

Journal of the American Chemical Society

Link to article, DOI:

$10.1021 / \mathrm{ja} 3011429$

Publication date:

2012

Document Version

Early version, also known as pre-print

Link back to DTU Orbit

Citation (APA):

Hatzakis, N. S., Wei, L., Jørgensen, S. K., Kunding, A. H., Bolinger, P-Y., Ehrlich, N., Makarov, I., Skjot, M., Svendsen, A., Hedegård, P., \& Stamou, D. (2012). Single Enzyme Studies Reveal the Existence of Discrete Functional States for Monomeric Enzymes and How They Are "Selected" upon Allosteric Regulation. Journal of the American Chemical Society, 134(22), 9296-9302. https://doi.org/10.1021/ja3011429

\section{General rights}

Copyright and moral rights for the publications made accessible in the public portal are retained by the authors and/or other copyright owners and it is a condition of accessing publications that users recognise and abide by the legal requirements associated with these rights.

- Users may download and print one copy of any publication from the public portal for the purpose of private study or research.

- You may not further distribute the material or use it for any profit-making activity or commercial gain

- You may freely distribute the URL identifying the publication in the public portal 


\section{Single Enzyme Studies Reveal the Existence of Discrete Functional States for Monomeric Enzymes and How They Are "Selected" upon Allosteric Regulation}

Nikos S. Hatzakis, ${ }^{\dagger}, \dagger, \S$ Li Wei, ${ }^{\dagger, t, \S}$ Sune K. Jorgensen, ${ }^{\dagger, \#}$ Andreas H. Kunding ${ }^{\dagger, \ddagger, \S}$

Pierre-Yves Bolinger, ${ }^{\dagger, \ddagger, \S, \nabla}$ Nicky Ehrlich, ${ }_{,}^{\dagger, \downarrow, \S}$ Ivan Makarov, ${ }^{\dagger, \ddagger, \S}$ Michael Skjot, ${ }^{\text {,l, }}$ Allan Svendsen, Per Hedegård, ${ }^{\perp}$ and Dimitrios Stamou, ${ }^{*, \neq, \downarrow, \S}$

${ }^{\dagger}$ Bio-Nanotechnology Laboratory, Department of Chemistry, Department of Neuroscience and Pharmacology, ${ }^{\star}$ Nano-Science Center, ${ }^{\S}$ Lundbeck Foundation Center Biomembranes in Nanomedicine, University of Copenhagen, 2100 Copenhagen, Denmark

${ }^{\|}$Novozymes A/S, Department of Protein Biochemistry, 2880 Bagsvaerd, Denmark

${ }^{\perp}$ Nano-Science Center, Niels Bohr Institute, University of Copenhagen, Denmark

Supporting Information

ABSTRACT: Allosteric regulation of enzymatic activity forms the basis for controlling a plethora of vital cellular processes. While the mechanism underlying regulation of multimeric enzymes is generally well understood and proposed to primarily operate via conformational selection, the mechanism underlying allosteric regulation of monomeric enzymes is poorly understood. Here we monitored for the first time allosteric regulation of enzymatic activity at the single molecule level. We measured single stochastic catalytic turnovers of a monomeric metabolic enzyme (Thermomyces lanuginosus

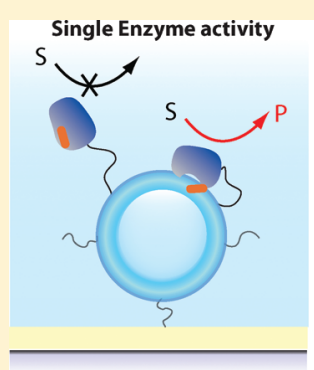

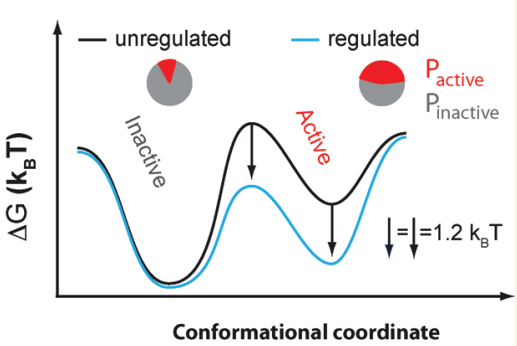
Lipase) while titrating its proximity to a lipid membrane that acts as an allosteric effector. The single molecule measurements revealed the existence of discrete binary functional states that could not be identified in macroscopic measurements due to ensemble averaging. The discrete functional states correlate with the enzyme's major conformational states and are redistributed in the presence of the regulatory effector. Thus, our data support allosteric regulation of monomeric enzymes to operate via selection of preexisting functional states and not via induction of new ones.

\section{INTRODUCTION}

Allosteric regulation of enzymatic activity is central for controlling a plethora of biological transformations vital for living organisms. The process of regulation is achieved through interactions with an effector, ligand or another protein, at a site distal from the active site. ${ }^{1-5} \mathrm{~A}$ central question, however, is how these effector interactions lead to regulation of enzymatic activity. To address this question, two competing models have been employed describing the regulation of multimeric enzymes. The KNF model proposes effector interactions on one enzyme binding-site to sequentially "induce" new conformational states on the rest of the monomers affecting their activity. ${ }^{6}$ The MWC model, on the other hand, proposes multimeric enzymes to undergo reversible transitions between discrete conformations assumed to have well-defined activity. Effector interactions are proposed to shift the equilibrium distribution by "selecting" one state with its corresponding activity. ${ }^{7}$

While the mechanism underlying regulation of multimeric enzymes is generally well described by these models, the mechanism underlying regulation of monomeric enzymes remains unclear. ${ }^{1,2,5,8}$ Current understanding primarily relies on bulk measurements that correlate changes in macroscopic activity with the redistribution of conformational equilibrium upon regulation. Bulk kinetics, however, cannot directly measure the inherent activity of each conformational state because they report the time-average macroscopic activity of an ensemble of enzymes. The existence of discrete functional states, their correlation to conformational states, and the putative selection or induction of a new functional state upon regulation could only be directly confirmed by single molecule studies. Thus, to date it remains unknown whether regulatory cofactors (a) redistribute a preexisting conformational equilibrium without changing the inherent activity of each conformational state, (b) induce a new conformational state with its corresponding inherent activity, or (c) operate via a convolution of both mechanisms a and $b$.

Here, to identify the mechanism underlying regulation of monomeric enzymes, we followed for the first time regulation

Received: February 11, 2012

Published: April 10, 2012 
of enzymatic catalysis at the single molecule level. We used measurements and statistical analysis of single catalytic events $^{9-17}$ to reveal the changes in the energy barriers along both the reaction and the regulatory coordinate, of a monomeric metabolic enzyme (Thermomyces lanuginosus lipase) whose activity we regulated in a quantitative and progressive manner by titrating its proximity to a lipid membrane. We identified the existence of discrete functional states that are linked to the enzyme's conformational states, and we found regulatory interactions to redistribute the probability to reside in these states but not to introduce new functional states. Our data thus support regulation of enzymatic activity to operate through selection and not via induction of new functional states, suggesting a mechanism akin to "conformational selection" (CS) rather than the "induced fit" (IF) hypothesis.

\section{RESULTS}

$T L L$ is a representative model of metabolic enzymes whose function is the degradation of fat. ${ }^{18}$ Similarly to other enzymes such as GTPases, ${ }^{19}$ kinases, ${ }^{4}$ proteases, ${ }^{20}$ and phospholipases, $^{21,22}$ TLL undergoes a conformational rearrangement upon regulation. When TLL interacts with its effector, a lipid membrane, the peptide-lid that otherwise blocks the active site is displaced, rendering TLL catalytically competent. ${ }^{23}$ This wellstudied conformational rearrangement is of regulatory nature and thus distinct from conformational interconversions along the reaction coordinate. ${ }^{24,25}$

As shown in Figure 1A, we coupled TLL on surface-tethered liposomes. ${ }^{26-29}$ Liposomes have been successfully employed to encapsulate and study single molecules. ${ }^{30}$ Here we employed these architectures as a scaffold bringing the enzyme in proximity to its effector ${ }^{23}$ while simultaneously minimizing nonspecific interactions with the solid surface that may alter its activity. To ensure a homogeneous, directional, immobilization of all enzymes, we site-specifically biotinylated a wellcharacterized single cysteine mutant of $T L L^{31}$ that was subsequently conjugated to a fluorescently labeled streptavidin and then tethered to the biotinylated liposomes (see Supporting Information (SI) Figures S1-3).

To locate enzymes and monitor single enzymatic turnovers in time, we used confocal fluorescent microscopy. ${ }^{9-17}$ Labeling fluorescently both the liposomes (DiD) and the enzymes (Alexa fluor 488) allowed us to measure the stoichiometry of the complexes and exclude from our measurements empty liposomes or enzymes bound nonspecifically to the surface (Figure $1 \mathrm{~B}$ and $\mathrm{C}$ ). We ensured that only one enzyme is immobilized per liposome by using a stoichiometry of $\sim 1: 20$, thus making it statistically unlikely $\left(P<10^{-3}\right)$ for a liposome to contain a second enzyme (SI Figure S4). To monitor the enzymatic activity, we employed the prefluorescent substrate of carboxy-fluorescein diacetate (CFDA) (SI Figures S5-6), which upon hydrolysis generates the highly fluorescent product carboxy-fluorescein (FAM). ${ }^{11}$ After addition of a saturating concentration of substrate (Figure S6), a single TLL immobilized on a liposome was positioned under the parked beam of the confocal microscope. Each enzymatic turnover produced a fluorescent burst before FAM diffused away from the confocal volume, providing the stochastic trajectory of single turnover events of Figure 1D. Positioning the laser on empty vesicles provided the background signal (Figure 1D, black trace). Special care was taken to ensure that (a) product release from enzyme was not rate limiting, (b) product did not
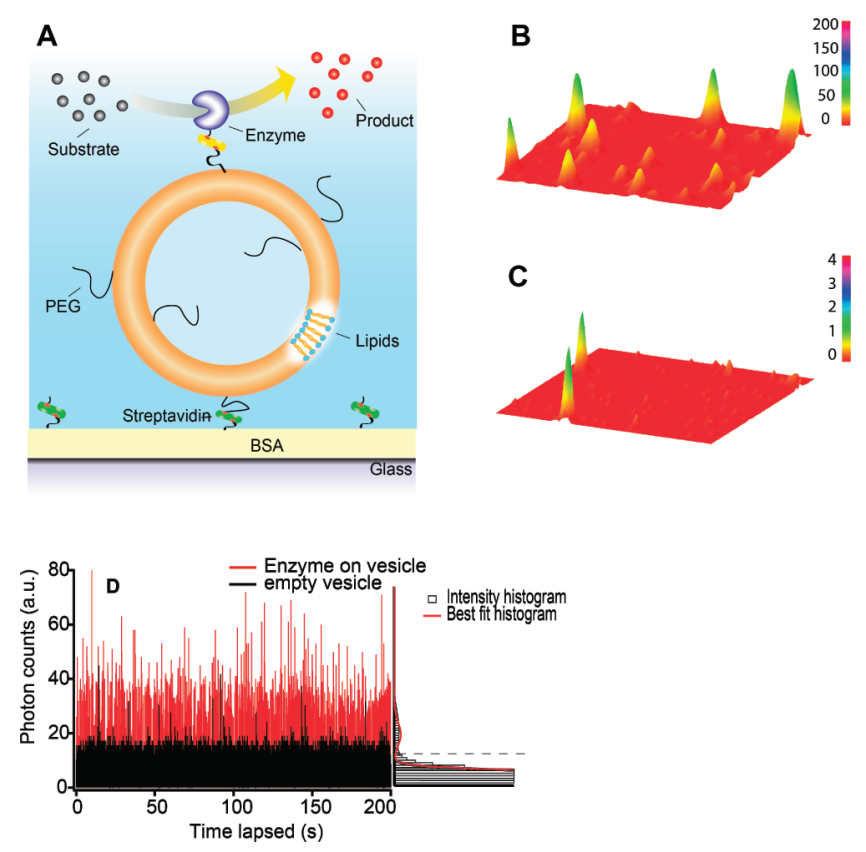

Figure 1. Assay to monitor membrane-regulated enzymatic processes at the single molecule level using fluorescent microscopy. (A) Schematic of the method. A single lipase (TLL) conjugated to a labeled streptavidin is coupled to liposomes through a flexible PEGbiotin linker. The liposomes are tethered on the glass coverslip passivated by streptavidin. A single prefluorescent substrate is enzymatically converted to the fluorescent product and is detected before it diffuses away from the confocal volume. (B, C) Zoom in a representative fluorescent micrograph showing respectively liposomes labeled with $\mathrm{DiD}$ and TLL enzymes labeled with Alexa fluor ${ }^{488}$. The low enzyme/liposome ratio ensures that only one enzyme is immobilized per liposome. (D) Time trace of product formation from a single $T L L$ (red trace). Each spike corresponds to single product formation. Control trace obtained from a liposome containing no enzyme for identical substrate concentration (black trace). No product accumulation on vesicle occurs. The detected photons are binned at $1 \mathrm{~ms}$ in both cases. The time trace histogram shown on the right-hand side of the trajectory is used to quantify the threshold between consecutive enzyme turnovers.

accumulate on the liposomes, and (c) product did not blink in the time scale of our measurements (see SI Figures S7-9).

Inspired by the conformational selection hypothesis, we employed a previously reported two-state statistical model ${ }^{32}$ to describe stochastic single-substrate turnovers; see the SI for detailed statistical analysis. A catalytic cycle begins when a substrate binds reversibly with the enzyme to form the enzyme-substrate complex. The complex yields the product with a rate $k_{a c t 1}$, and the enzyme rapidly recovers to the original state. Once in the initial state, the enzyme either performs another catalytic cycle or interconverts to a second conformational state with different inherent activity $\left(k_{\text {act } 2}\right)$ (green enzyme in Figure 2A). At saturating substrate concentrations where $k_{\text {act }}$ becomes the rate-limiting step, the probability density of the waiting time $\left(p_{w}\right)$ between two adjacent turnover cycles can be described by the following:

$$
P w(t)=\frac{1+\nu}{2} \lambda_{+} \mathrm{e}^{-\lambda_{+} t}+\frac{1-\nu}{2} \lambda_{-} \mathrm{e}^{-\lambda_{-} t}
$$

where 
A
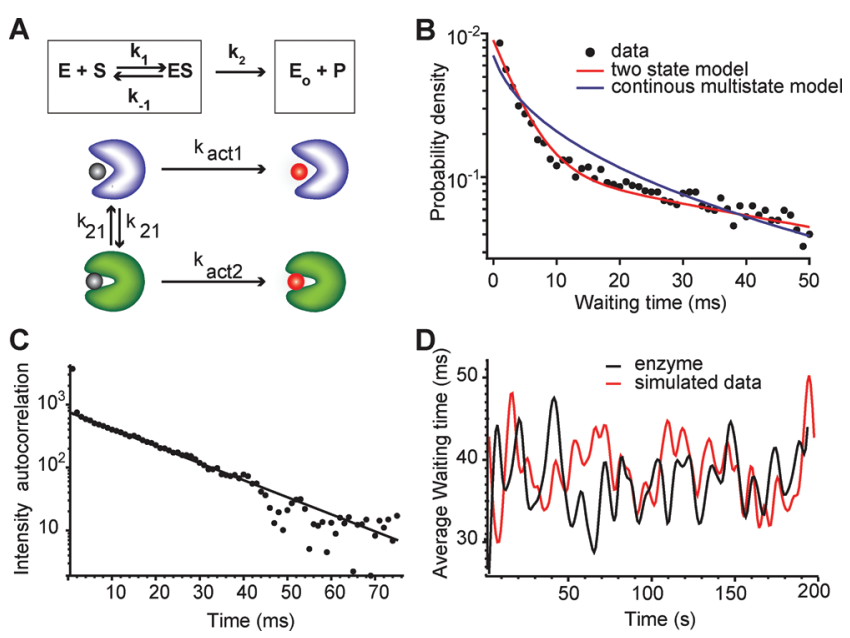

Figure 2. Two-state model describing well the stochastic activity pattern of TLL. (A) Schematic representation of a general two-state model employed to describe the catalytic behavior of enzymes. The model is an extension on the Michaelis-Menten equation containing two activity states $\left(A_{1}\right.$ and $\left.A_{2}\right)$ with respective activity rates $k_{\text {act } 1}$ and $k_{a c t 2}$. (B) Histogram of the waiting time between consecutive turnovers of TLL obtained at saturating conditions plotted on a log-linear scale (black dots) (see SI Figure S23 for all data). The red line is the double exponential decay (see eq 1 ) that derives from the two-state model. The blue line is the fitting obtained with a stretched exponential decay widely employed to fit distributions resulting from multiple reaction rates. A substantial improved fitting is observed with the two-state model. (C) Intensity autocorrelation of TLL plotted on a log-linear scale. $C_{s}(t)$ decays monoexponentially with an exponent that matches the sum of the interconversion rates in agreement with the presence of two discrete activity states. (D) (Black trace) Running average of TLL waiting time in a moving window with a width of $\sim 2 \mathrm{~s}$. TLL's average activity is not constant but fluctuates in all time scales around an average value as shown for multiple single enzymes. (Red trace) Time trace of average $T L L$ waiting time as simulated by using the parameters extracted by the two-activity states model. An enzyme oscillating between two activity states in the millisecond time scale may exhibit activity fluctuations occurring in slower (sec) time scales. Thus, dynamic disorder is not a prerequisite of multiple activity states.

$$
\begin{aligned}
\lambda_{ \pm}= & \frac{k_{a c t 1}+k_{12}+k_{a c t 2}+k_{21}}{2} \\
& \pm \sqrt{\left(\frac{k_{a c t 1}+k_{12}-k_{a c t 2}-k_{21}}{2}\right)^{2}+k_{21} k_{12}}
\end{aligned}
$$

and

$$
\frac{1}{k_{2}}=\frac{1+v}{2 \lambda_{+}}+\frac{1-v}{2 \lambda_{-}}
$$

the average activity of a single enzyme is given by

$$
k_{2}=P_{a c t 1} k_{a c t 1}+\left(1-P_{a c t 1}\right) k_{a c t 2}
$$

where

$$
P_{a c t 1}=\frac{k_{21}}{k_{21}+k_{12}}
$$

To examine whether the two-state model describes well the activity of $T L L$, we analyzed $p_{w}$, the enzyme's contribution to the intensity autocorrelation $\left(C_{s}(\tau)\right), C_{s}(\tau)=$ $\left.\langle I s(t)\rangle^{2}\left[\left(k_{12} k_{21}\left(k_{a c t 1}-k_{a c t 2}\right)^{2}\right) /\left(k_{a c t 1} k_{21}-k_{a c t 2} k_{12}\right)^{2}\right)\right] e-\left(k_{12}\right.$ $\left.+k_{21}\right) \tau$, and the autocorrelation of waiting times $\left(C_{n}\right) C_{n}=$ $\left(\left\langle\tau_{n} \tau_{1}\right\rangle-\left\langle\tau_{1}\right\rangle^{2}\right) /\left(\left\langle\tau_{1}^{2}\right\rangle-\left\langle\tau_{1}\right\rangle^{2}\right)$. Similar to earlier observations recorded at saturating conditions, we obtained a nonexponential decay of the waiting time distribution, a behavior previously attributed to multiple reaction rates. ${ }^{9-15}$ Interestingly, we found the double exponential of eq 1 to fit accurately the experimental data (see Figure 2B and SI Figure S23 for full data), and furthermore so, significantly better than the stretched exponential decay previously employed to describe continuous multistate activity models (see SI for detailed fitting and comparison between the two models). Additionally both $C_{s}(t)$ and $C_{n}$ showed a monoexponential decay (Figure 2C and Figures S14-15, see SI for full description), consistent with the presence of two functional states. ${ }^{9}$

Next we examined whether additional enzymes possess a discrete number of functional states. We obtained published activity traces for $\beta$-galactosidase from E. coli, ${ }^{9}$ for the Nitrite reductase (NR) from Alcaligenes faecalis, ${ }^{10}$ and for bovine chymotrypsin, ${ }^{12}$ and we recorded the activity of the non-bilayer regulated Lipase from Candida Antarctica (CALB). In agreement with previous findings, $\beta$-galactosidase and chymotrypsin could not be fitted with a two-state model (see SI Figure S26). ${ }^{9,12}$ This is expected for $\beta$-galactosidase, which is composed of four monomers that could catalyze independently of each other. We found however both NR and CALB, see SI and Figures S23-25, to exhibit two discrete activity states in agreement with TLL. Out of the four monomeric enzymes reexamined here, three exhibited behavior that could be explained by two activity state model which may raise questions on the interpretation of single enzymes studies supporting the existence of multiple activity states. ${ }^{9-15}$ The two-state model permitted us to quantify the inherent activity of discrete states instead of a distribution of activities ${ }^{9-15}$ (see Table S2). This was achieved by combining information from $p_{w}$ and $C_{n}$ (see SI for statistical analysis and Figures S12-14). We found TLL to interconvert between states of significantly different inherent activities $k_{\text {act } 1}=0.23 \pm 0.03 \mathrm{~ms}^{-1}$ and $k_{\text {act } 2}=0.0125 \pm 0.003$ $\mathrm{ms}^{-1}$. Since $k_{\text {act } 2}$ is on the average $\sim 20$ times smaller than $k_{\text {act } 1}$, it can be approximated as practically zero and the binary enzymatic states can be described as digital-like, i.e. an active and a practically inactive state (see SI Table S3 and Figure S19). These functional states correlate well with the two major conformational states of TLL, since the inactive state can be assigned to the closed lid conformation while the highly active state to the open lid conformation. ${ }^{33,34}$ It is noteworthy that the lid dynamics in TLL are not taking place along the reaction coordinate, as recently shown for other enzymes; ${ }^{24,25}$ in the open state, the enzyme's active site is substrate accessible, and multiple turnovers may occur. 4,35

Next we calculated the interconversion rates between the two functional states. The extracted kinetic constants $k_{12}=0.062$ $\mathrm{ms}^{-1}$ and $k_{21}=0.003 \mathrm{~ms}^{-1}$ are in agreement with earlier studies on protein dynamics showing domain motions occurring on the microsecond to millisecond time scale, ${ }^{24}$ supporting the link between functional and structural states. As a consistency check, we examined $C_{s}(t)$, which for the two activity states model should decay monoexponentially (Figure 2C) with an exponent that equals the sum of the two interconversion rates. ${ }^{9,32}$ Indeed, the exponent of the intensity autocorrelation decay $K_{(\mathrm{I})}=0.06 \pm 0.001 \mathrm{~ms}^{-1}$ equals the sum of interconversion rates $k_{12}+k_{21}=0.065 \pm 0.03 \mathrm{~ms}^{-1}$ (see SI Figure S15 and Table S2). An alternative method employed to quantify activity fluctuations is the activity autocorrelation $K(t) .{ }^{9}$ We found, however, the time dependence of $K(t)$ to strongly depend on the averaging function used to calculate $k_{2}$, 
thus not providing any further information not encoded in the intensity autocorrelation function $\operatorname{Cs}(t)$ (see SI for detailed statistical analysis).

Plotting the time average of the waiting time between events revealed that the millisecond activity fluctuations of TLL extend to the second time scales (Figure 2D). Activity oscillations in multiple time scales (millisecond to second) were observed previously and were interpreted to be the consequence of a wide distribution of activity rates, ${ }^{9-15}$ we thus next examined whether they may also result from a two-state system. Indeed, when we simulated the waiting time using the parameters extracted from fitting our data, we observed activity fluctuations very similar to the experimental ones in terms of mean value, time scale, and amplitude (Figure 2D and SI Figures S16-18). Thus, activity fluctuations in multiple time scales may well originate from stochastic oscillations between two activity states. $^{2}$

Recent studies ${ }^{36}$ proposed the static heterogeneity of activity between individual enzymes to originate from very slow interconversion between conformational states of different activity; albeit, such transitions were not recorded. Within the time scale of our measurements and for the number of interrogated enzymes, we also did not observe such transitions. If, however, a transition to a new long-lived activity state would occur, it would result in a new set of the four rates $\left(k_{\text {act } 1}, k_{\text {act } 2}\right.$, $k_{12}$, and $k_{21}$ ) and thus to a systematic shift of the activity oscillations around a new average value.

A basic consequence of our findings is that in the limiting case of digital-like activity (i.e. when $k_{\text {act } 2} \ll k_{a c t 1}$, which can be approximated as $\left.k_{\text {act } 2} \rightarrow 0\right), k_{2}$ as measured by ensemble measurements and single molecule experiments would be the product of the enzyme's probability to reside on the highly active state $\left(P_{a c t}\right)$ and the inherent activity of the active state $\left(k_{\text {act } 1}\right)$.

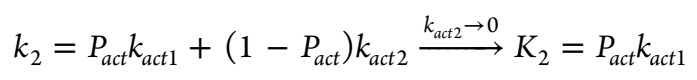

The compelling question we addressed next is whether allosteric regulation of $k_{2}$ proceeds via redistribution of the equilibrium between states, thus changing $P_{a c t}$, as the CS hypothesis posits, by introduction of a new highly active state, thus altering $k_{\text {actl }}$, as the IF hypothesis posits, or by a convolution of both. Hence, we monitored the activity of single $T L L$ while we modulated systematically its access to the effector-bilayer. TLL is an interfacially activated enzyme, and its activity is allosterically regulated by the bilayer. In solution the enzyme's active site is blocked by the peptide-lid. Upon interaction with a bilayer, TLL undergoes a major conformational rearrangement, and the lid rotates around its hinge facilitating binding to the bilayer and exposing the enzymes active site. $^{23}$ This conformational rearrangement allows substrate access to the active site and renders the enzyme in a catalytically competent form. One way to modulate TLL's access to its effector is to sterically hinder its access to the bilayer. Alternatively the same could be achieved by altering the length of the tether. Here we choose to modulate the enzymes access to its effector-bilayer by using the well established methodology of imposing steric hindrance. ${ }^{37}$ By gradually increasing the percentage of a polyethylene glycol (PEG modified phospholipid in the liposome-scaffold (Figure $3 \mathrm{~A}$ and $\mathrm{B}$ and SI Figure S20) between 0 and $2.1 \%$ molar, we gradually restricted the enzyme's access to the bilayer. In a control experiment we verified that PEG does not interfere directly
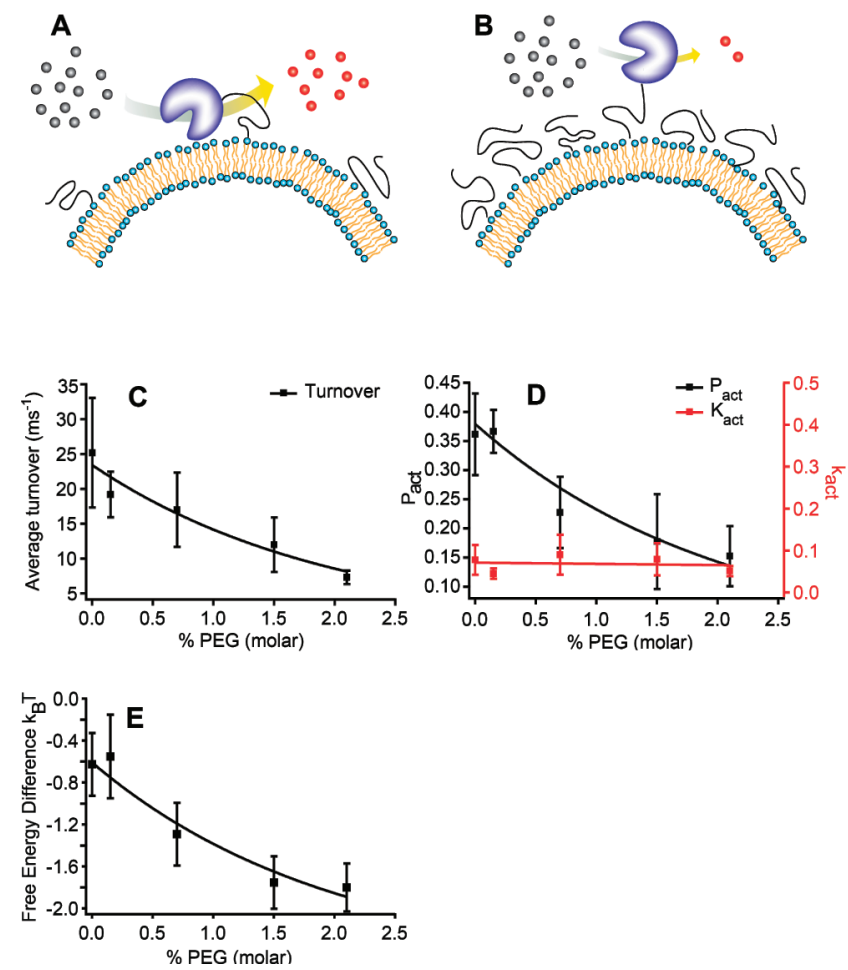

Figure 3. Mechanistic origin of lipase activity regulation. (A, B) Schematic representation (not to scale) of the experimental set up employed to control in a systematic fashion TLL's accessibility to the effector-bilayer. (A) At low PEG phospholipids concentration TLL has unlimited access to the bilayer. (B) At high PEG concentrations the bilayer is highly hindered. (C) Incrementing the PEG concentration on liposomes results in a progressive $\sim 4$-fold exponential decrease of the enzymatic reaction rate, in good agreement with the probability of the enzyme to access the bilayer (see SI). Each data point is the average value of at least four individual single enzyme experiments (see SI Table S1). (D) Deconvolution of the parameters that govern TLL activity regulation. Independently of the PEG concentration, the enzyme exhibits a constant within uncertainty activity $k_{\text {act } 1}$. Restricting the enzyme's accessibility to the bilayer results in exponential decay of $P_{a c t}$. Thus, enzyme's regulation does not occur via an increased inherent enzymatic activity $\left(k_{a c t 1}\right)$ but rather predominantly depends on increased probability of residing in active states $\left(P_{a c t}\right)$. (E) Origin of energy landscape stabilization upon interaction with the effector. Incrementing the enzyme's accessibility to the bilayers progressively confers a relative energetic stabilization of the active state by $1.2 k_{\mathrm{B}} T$. Error bars in parts $\mathrm{C}, \mathrm{D}$, and $\mathrm{E}$ represent the standard deviation for different single molecules.

with enzymatic activity (see SI Figures S10 and 11). At 2.1\% molar PEG we found TLL to exhibit a residual activity rate of $\sim 7 \mathrm{~s}^{-1}$ (Figure 3C). Increasing enzyme's access to the bilayer resulted in a $\sim 4$-fold exponential increase of activity to a maximum of $\sim 26 \mathrm{~s}^{-1}$, in good agreement with bulk measurements $^{23}$ (see SI Figures S10-11).

Furthermore, we found $P_{a c t}$ to depend strongly on the regulatory effect of the bilayer (Figure 3D). Systematically hindering bilayer-access progressively shifted the equilibrium of active/inactive states, reducing $P_{\text {act }}$ from $\sim 0.4$ to $\sim 0.15$. The reduction of $P_{\text {act }}$ explains entirely the change in $k_{2}$ (Figure 3C) and can be fitted well with the calculated, exponentially decaying, probability of a protein to reach a PEG-covered surface $^{37}$ (see also SI). Interestingly, Figure 3D reveals that for all degrees of regulation TLL molecules were found to have constant $k_{\text {actl }}$ (inherent activity) within error; see SI for all data. 
These data thus provide direct evidence that regulatory interactions do not introduce a new functional state, as a mechanism based on induced fit would premise.

To analyze quantitatively the changes in the free energy $(\Delta G)$ of TLL upon regulation, we used the microscopic rate constants (see Table S1) and the Arrhenius equation $(\Delta G=$ $\left.-k_{\mathrm{B}} T \ln \left(k_{12} / k_{21}\right)\right)$. Increasing bilayer-access was found to decrease progressively the $\Delta G$ of the two states in favor of the active state to a final value of $1.2 k_{\mathrm{B}} T$, which is in line with earlier studies on protein dynamics ${ }^{24}$ (see Figure 3E and SI Figure S21). Next we plotted $k_{12}$ and $k_{21}$ as a function of PEG concentration (SI Figure S21). We found a strong dependency on bilayer accessibility for $k_{21}$ that was present despite the large inhomogeneity in the rates, while $k_{12}$ remained constant within error. Because the inactive state corresponds to a closed-lid state obtained for the enzyme residing in solution, its energy level should be independent of accessing the bilayer and can thus be taken as a reference point in our energy landscape (Figure 4). Under this assumption, we conclude that regulation

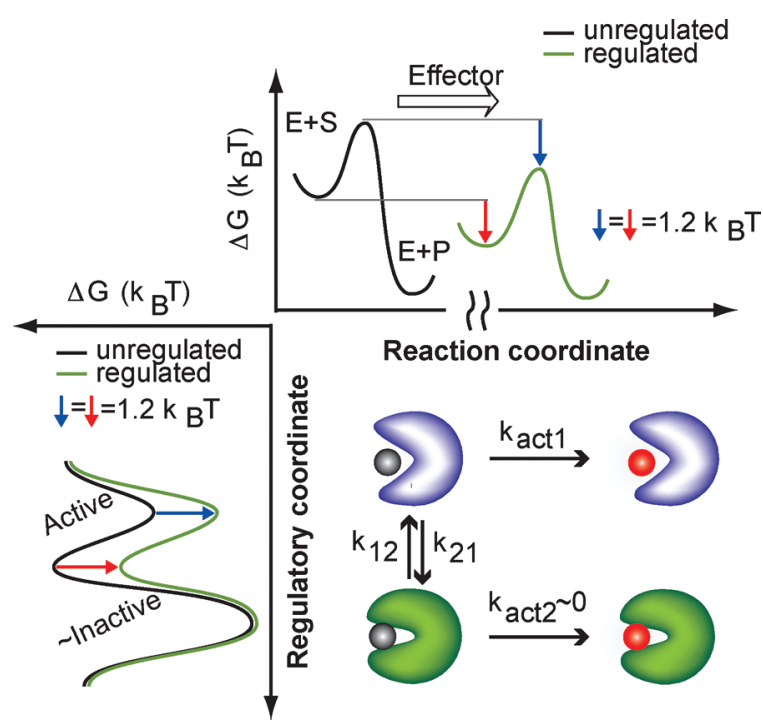

Figure 4. Changes in the free energy landscape of TLL upon regulation. Quantification of the effect of regulatory interactions on the energy landscape of both the regulatory coordinate (vertical axis) and the reaction coordinate (horizontal axis) of TLL. Effector interactions remodel the regulatory coordinate by equal energetic stabilization of the active state and the energy barrier to the active state by $1.2 k_{\mathrm{B}} T$ (green versus black one-dimensional cross section of the energy landscape), thus redistributing the equilibrium and increasing the probability to reside on the highly active state (shown as blue in the cartoon inset). Regulation also energetically favors the transition state to the product formation by $1.2 k_{\mathrm{B}} T$, thus maintaining a constant inherent activity of the highly active state independent of regulatory interactions.

of TLL is achieved by simultaneous and equal energetic stabilization of the active state and of the energy barrier between the two states (Figure 4, regulatory coordinate) ${ }^{5,38}$ An additional control experiment would be to introduce additional mutations that shift the conformational equilibrium toward one of the states. ${ }^{39}$ However, these mutations would also alter the enzyme's energy landscape, prohibiting us from directly comparing the free energy difference between the two variants. The fact that $k_{\text {act } 1}$ remained constant (Figure 3D) independent of bilayer accessibility suggests that the presence of the effector stabilizes equally the active state and the reaction transition energy (Figure 4, reaction coordinate).

\section{CONCLUSIONS}

Historically, selective stabilization between preexisting conformational states was proposed to explain the allosteric regulation of multisubunit enzymes and proteins, such as aspartate transcarboamylase and hemoglobin. ${ }^{1}$ Later studies, both in bulk and with single molecule resolution, proposed monomeric proteins to operate through conformational selection. ${ }^{3,38,40-45}$ The same mechanism was successfully borrowed for monomeric enzymes to explain substrate mediated conformational redistributions along the reaction coordinate. ${ }^{2,5,25,46}$

Regulated enzymes, however, in addition to their reaction coordinate, have a conformational-regulatory coordinate. A major structural rearrangement has to occur for the enzyme to adopt the active conformational state, where the active site is accessible and catalysis may occur. ${ }^{4,21,47}$ Indeed, the existence of discrete active and inactive conformational states has been documented for multiple monomeric regulated enzymes. $^{4,21,35,47}$ However, to date there is limited knowledge whether effectors simply redistribute the equilibrium of preexisting conformational states, ${ }^{35,47,48}$ introduce a new conformational state, ${ }^{49}$ or a convolution of the above. In either case, the effect of allosteric interactions on the enzymatic reaction coordinate (see Figure 4) and thus on the enzyme's functional states cannot be quantified by experiments in bulk due to ensemble averaging.

Studies on enzymatic activity regulation are based primarily on structural evidence, and it is intuitively assumed that each enzymatic major conformational state has a well-defined activity. However, all single molecule activity measurements on nonregulated enzymes have instead provided evidence, and interpretations thereof, supporting the existence of a continuous distribution of activity states. ${ }^{9-15}$ This putative absence of discrete functional states indicates that the intuitive, albeit nonvalidated, assumption of one to one correspondence between conformational and functional states may not hold. To resolve this problem, we decided to look for the effect of regulation on functional states directly, arguing in addition that, in the case of regulation, function is a more important observable compared to structure. In that sense, here we are discussing "functional selection" instead of "conformational selection".

To test for the existence of discrete functional states, we employed single enzyme kinetics, ${ }^{9-15}$ since their presence would be masked in experiments in bulk. One central finding of this work that sets the foundation for unraveling the mechanism of enzymatic activity regulation is the identification of discrete functional states, which does not any longer preclude selection as a regulatory mechanism. The presence of two functional states furthermore supports the simplification of two state models employed by ensemble techniques such as NMR to describe protein behavior.

In this study to monitor allosteric regulation of enzymatic activity with single molecule resolution, we have employed a novel platform based on arrays of surface tethered liposomes. $^{26-29}$ Liposomes constitute an ideal biocompatible scaffold to spatially confine practically any biocatalyst minimizing denaturating interactions with hard matter that may alter its function and permit reproducible measurements of single enzyme kinetics. This generic platform can thus be 
extended to investigate protein ligand or protein membrane interactions as well as the behavior of regulated or not enzymes with single molecule resolution. Titrating, for example, the concentration of the water-soluble small molecule effector would allow studying the mechanism of allosteric regulation of nonbilayer dependent enzymes. Altering bilayer properties such as lipid charge, composition, and phase state would permit investigating their effect on the behavior of bilayer-regulated enzymes. Here we used this platform to titrate the enzymes' proximity to its effector-bilayer and decipher the mechanism of allosteric regulation using single molecule experiments.

Our single molecule findings extending beyond the reaction coordinate allowed us to quantify the free energy landscape of allosteric regulation of enzymatic activity. Besides calculating energy barriers along the reaction coordinate, ${ }^{24,25,50}$ we quantified for the first time energy barriers along the regulatory coordinate. ${ }^{4,40,51,52} \mathrm{We}$ found regulatory interactions to equally stabilize the highly active functional state and the reaction transition energy. These findings thus demonstrate regulation of TLL activity to operate via selection of preexisting functional states. Since we did not measure the induction of new functional states, our data support the prevalence of a mechanism akin to "conformational selection" over the "induced fit" hypothesis.

The actual activity ratio $\left(R_{A}\right)$ of the two activity states of TLL was $\sim 20$, suggesting they could indeed form the basis of an intramolecular binary switch of activity. $R_{A}$ plays a critical role in regulation, since it poses the upper limit to the efficiency of the process $\left(E_{\max }=1-1 / R_{A}\right)$ that is reached for $100 \%$ efficient conformational selection. Obviously if $R_{A}=1$, regulation of activity through conformational selection is not feasible $\left(E_{\max }=\right.$ $0)$. For $T L L, E_{\max }=0.95$, making it a candidate for digital-like regulation; ${ }^{23}$ however, under in vitro conditions, a less than perfect regulation efficiency $\left(E_{R}=\left(P_{a c t(\max )}-P_{a c t(\min )}\right) / P_{\text {act }(\max )}\right.$ $=0.77)$ resulted in an overall efficiency $E_{T}=E_{\max } E_{R}=0.73$.

Out of a total of seven enzymes measured with single turnover resolution to date, ${ }^{9-15}$ we screened five, four of which where monomeric (including TLL; see SI), for the presence of discrete functional states. Three of the monomeric enzymes could be fitted with a two-state model and are thus, in principle, compatible with a mechanism akin to conformational selection. The existence of a minimum number of functional states is consistent with bulk findings on regulated proteins, thus bringing into line single molecule work with ensemble studies. Further measurements will reveal whether redistribution between discrete preexisting functional states is a unifying mechanism or whether other monomeric enzymes operate through more complex regulatory principles.

Providing insights on the determinants of enzymatic regulation is essential both for understanding cellular processes and for the design of novel biocatalysts with tailor-made functionalities.

\section{ASSOCIATED CONTENT}

\section{S Supporting Information}

Experimental procedure and controls in Supporting Methods, model development and statistical analysis in Supporting Text, and Figures S1-26 and Tables S1-3 as described in the paper. This material is available free of charge via the Internet at http://pubs.acs.org.

\section{AUTHOR INFORMATION}

\section{Corresponding Author}

Stamou@nano.ku.dk; Hatzakis@nano.ku.dk

\section{Present Addresses}

"Department of Chemistry, University of Copenhagen, Denmark.

${ }^{\nabla}$ Credit Suisse, Uetlibergstrasse 231, 8045 Zürich ZH, Switzerland.

ONovo Nordisk A/S Novo Nordisk Park DK-2760 Måløv, Denmark.

\section{Author Contributions}

N.S.H and L.W. contributed equally.

\section{Notes}

The authors declare no competing financial interest.

\section{ACKNOWLEDGMENTS}

We thank Prof. T. Bjornholm for critically reading the manuscript, Prof. J. Hofkens and Dr. J. Grandl for fruitful discussions, and Prof. S. X. Xie, Prof. T. J. Aartsma, and Prof. G. W. Canters for providing experimental data for retreatment. This work was supported by the Danish Council for Independent and Strategic Research, the Lundbeck Foundation, and the University of Copenhagen program of excellence "Single molecule Nanoscience" and "UNIK-Synthetic Biology".

\section{REFERENCES}

(1) Changeux, J. P.; Edelstein, S. J. Science 2005, 308, 1424.

(2) Boehr, D. D.; Nussinov, R.; Wright, P. E. Nat. Chem. Biol. 2009, $5,789$.

(3) Tzeng, S. R.; Kalodimos, C. G. Nature 2009, 462, 368.

(4) Kuriyan, J.; Eisenberg, D. Nature 2007, 450, 983.

(5) Goodey, N. M.; Benkovic, S. J. Nat. Chem. Biol. 2008, 4, 474.

(6) Koshland, D. E.; Nemethy, G.; Filmer, D. Biochemistry (Moscow) $1966,5,365$.

(7) Monod, J.; Wyman, J.; Changeux, J. P. J. Mol. Biol. 1965, 12, 88.

(8) Ma, B. Y.; Nussinov, R. Curr. Opin. Chem. Biol. 2010, 14, 652.

(9) English, B. P.; Min, W.; van Oijen, A. M.; Lee, K. T.; Luo, G. B.; Sun, H. Y.; Cherayil, B. J.; Kou, S. C.; Xie, X. S. Nat. Chem. Biol. 2006, 2, 87.

(10) Kuznetsova, S.; Zauner, G.; Aartsma, T. J.; Engelkamp, H.; Hatzakis, N.; Rowan, A. E.; Nolte, R. J. M.; Christianen, P. C. M.; Canters, G. W. Proc. Natl. Acad. Sci. U. S. A. 2008, 105, 3250.

(11) Hatzakis, N. S.; Engelkamp, H.; Velonia, K.; Hofkens, J.; Christianen, P. C. M.; Svendsen, A.; Patkar, S. A.; Vind, J.; Maan, J. C.; Rowan, A. E.; Nolte, R. J. M. Chem. Commun. (Cambridge) 2006, 2012.

(12) De Cremer, G.; Roeffaers, M. B. J.; Baruah, M.; Sliwa, M.; Sels, B. F.; Hofkens, J.; De Vos, D. E. J. Am. Chem. Soc. 2007, 129, 15458.

(13) Flomenbom, O.; Velonia, K.; Loos, D.; Masuo, S.; Cotlet, M.; Engelborghs, Y.; Hofkens, J.; Rowan, A. E.; Nolte, R. J. M.; Van der Auweraer, M.; de Schryver, F. C.; Klafter, J. Proc. Natl. Acad. Sci. U. S. A. 2005, 102, 2368.

(14) Lu, H. P.; Xun, L. Y.; Xie, X. S. Science 1998, 282, 1877.

(15) Edman, L.; Foldes-Papp, Z.; Wennmalm, S.; Rigler, R. Chem. Phys. 1999, 247, 11.

(16) Claessen, V. I.; Engelkamp, H.; Christianen, P. C. M.; Maan, J. C.; Nolte, R. J. M.; Blank, K.; Rowan, A. E. In Annual Review of Analytical Chemistry, Vol 3; Yeung, E. S., Zare, R. N., Eds.; Annual Reviews: Palo Alto, CA, 2010; Vol. 3, p 319.

(17) Engelkamp, H.; Hatzakis, N. S.; Hofkens, J.; De Schryver, F. C.; Nolte, R. J. M.; Rowan, A. E. Chem. Commun. (Cambridge) 2006, 935.

(18) Shi, Y. G.; Burn, P. Nat. Rev. Drug Discovery 2004, 3, 695.

(19) Vetter, I. R.; Wittinghofer, A. Science 2001, 294, 1299. 
(20) Wolan, D. W.; Zorn, J. A.; Gray, D. C.; Wells, J. A. Science 2009, $326,853$.

(21) Lyon, A. M.; Tesmer, V. M.; Dhamsania, V. D.; Thal, D. M.; Gutierrez, J.; Chowdhury, S.; Suddala, K. C.; Northup, J. K.; Tesmer, J. J. G. Nat. Struct. Mol. Biol. 2011, 18, 999.

(22) Gudmand, M.; Rocha, S.; Hatzakis, N. S.; Peneva, K.; Mullen, K.; Stamou, D.; Uji-I, H.; Hofkens, J.; Bjornholm, T.; Heimburg, T. Biophys. J. 2010, 98, 1873.

(23) Cajal, Y.; Svendsen, A.; Girona, V.; Patkar, S. A.; Alsina, M. A. Biochemistry (Moscow) 2000, 39, 413.

(24) Henzler-Wildman, K. A.; Thai, V.; Lei, M.; Ott, M.; Wolf-Watz, M.; Fenn, T.; Pozharski, E.; Wilson, M. A.; Petsko, G. A.; Karplus, M.; Hubner, C. G.; Kern, D. Nature 2007, 450, 838.

(25) Boehr, D. D.; McElheny, D.; Dyson, H. J.; Wright, P. E. Science 2006, 313, 1638.

(26) Hatzakis, N. S.; Bhatia, V. K.; Larsen, J.; Madsen, K. L.; Bolinger, P. Y.; Kunding, A. H.; Castillo, J.; Gether, U.; Hedegard, P.; Stamou, D. Nat. Chem. Biol. 2009, 5, 835.

(27) Bendix, P. M.; Pedersen, M. S.; Stamou, D. Proc. Natl. Acad. Sci. U. S. A. 2009, 106, 12341.

(28) Larsen, J.; Hatzakis, N. S.; Stamou, D. J. Am. Chem. Soc. 2011, $133,10685$.

(29) Christensen, S. M.; Bolinger, P.-Y.; Hatzakis, N. S.; Mortensen, M. W.; Stamou, D. Nat. Nano 2012, 7, 51.

(30) Cisse, I.; Okumus, B.; Joo, C.; Ha, T. J. Proc. Natl. Acad. Sci. U. S. A. 2007, 104, 12646.

(31) Svendsen, A.; Clausen, I. G.; Patkar, S. A.; Borch, K.; Thellersen, M. Lipases, Part A; Elsevier Academic Press Inc: San Diego, CA, 1997; Vol. 284, p 317.

(32) Kou, S. C.; Cherayil, B. J.; Min, W.; English, B. P.; Xie, X. S. J. Phys. Chem. B 2005, 109, 19068.

(33) Lawson, D. M.; Brzozowski, A. M.; Dodson, G. G.; Hubbard, R. E.; Huge-Jensen, B.; Boel, E.; Derewenda, Z. S. Lipases: Their Structure, Biochemistry and Application; Cambridge University Press: 1994.

(34) Lawson, D. M.; Brzozowski, A. M.; Rety, S.; Verma, C.; Dodson, G. G. Protein Eng. 1994, 7, 543.

(35) Choi, Y.; Moody, I. S.; Sims, P. C.; Hunt, S. R.; Corso, B. L.; Perez, I.; Weiss, G. A.; Collins, P. G. Science 2012, 335, 319.

(36) Rissin, D. M.; Gorris, H. H.; Walt, D. R. J. Am. Chem. Soc. 2008, 130,5349 .

(37) NopplSimson, D. A.; Needham, D. Biophys. J. 1996, 70, 1391.

(38) Henzler-Wildman, K. A.; Lei, M.; Thai, V.; Kerns, S. J.; Karplus, M.; Kern, D. Nature 2007, 450, 913.

(39) Peters, G. H.; vanAalten, D. M. F.; Svendsen, A.; Bywater, R. Protein Eng. 1997, 10, 149.

(40) Zhao, Y. F.; Terry, D.; Shi, L.; Weinstein, H.; Blanchard, S. C.; Javitch, J. A. Nature 2010, 465, 188.

(41) Bokoch, M. P.; Zou, Y. Z.; Rasmussen, S. G. F.; Liu, C. W.; Nygaard, R.; Rosenbaum, D. M.; Fung, J. J.; Choi, H. J.; Thian, F. S.; Kobilka, T. S.; Puglisi, J. D.; Weis, W. I.; Pardo, L.; Prosser, R. S.; Mueller, L.; Kobilka, B. K. Nature 2010, 463, 108.

(42) Popovych, N.; Sun, S. J.; Ebright, R. H.; Kalodimos, C. G. Nat. Struct. Mol. Biol. 2006, 13, 831.

(43) Zhou, R. B.; Kunzelmann, S.; Webb, M. R.; Ha, T. Nano Lett. 2011, 11, 5482.

(44) Hohng, S.; Zhou, R. B.; Nahas, M. K.; Yu, J.; Schulten, K.; Lilley,

D. M. J.; Ha, T. J. Science 2007, 318, 279.

(45) Grashoff, C.; Hoffman, B. D.; Brenner, M. D.; Zhou, R. B.; Parsons, M.; Yang, M. T.; McLean, M. A.; Sligar, S. G.; Chen, C. S.; Ha, T.; Schwartz, M. A. Nature 2010, 466, 263.

(46) Hammes, G. G.; Chang, Y. C.; Oas, T. G. Proc. Natl. Acad. Sci. U. S. A. 2009, 106, 13737.

(47) Masterson, L. R; Cheng, C.; Yu, T.; Tonelli, M.; Kornev, A.; Taylor, S. S.; Veglia, G. Nat. Chem. Biol. 2010, 6, 821.

(48) Bakan, A.; Bahar, I. Proc. Natl. Acad. Sci. U. S. A. 2009, 106, 14349.

(49) Kadirvelraj, R.; Sennett, N. C.; Polizzi, S. J.; Weitzel, S.; Wood, Z. A. Biochemistry 2011, 50, 5780 .
(50) Pisliakov, A. V.; Cao, J.; Kamerlin, S. C. L.; Warshel, A. Proc. Natl. Acad. Sci. U. S. A. 2009, 106, 17359.

(51) Cornish, P. V.; Ermolenko, D. N.; Staple, D. W.; Hoang, L.; Hickerson, R. P.; Noller, H. F.; Ha, T. Proc. Natl. Acad. Sci. U. S. A. 2009, 106, 2571.

(52) Aitken, C. E.; Puglisi, J. D. Nat. Struct. Mol. Biol. 2010, 17, 793. 